\title{
ON THE NUMERICAL SOLUTION OF HEAT CONDUCTION PROBLEMS IN TWO AND THREE SPACE VARIABLES
}

\author{
BY \\ JIM DOUGLAS, JR., AND H. H. RACHFORD, JR.
}

1. Introduction. Many practical heat conduction questions lead to problems not conveniently solvable by classical methods, such as separation of variables techniques or the use of Green's functions. As a result, quite a number of procedures for obtaining approximate solutions have been proposed. It is the objective of this paper to introduce a new finite difference method applicable directly to unsteady-state (parabolic) problems in either two or three space variables and indirectly as an iteration technique to steady-state (elliptic) problems using the time step as a parameter.

The procedure to be discussed is an implicit method that, for the purpose of calculation, can be factored into a multi-stage process to step ahead one time increment in such a manner that the solution of the linear equations arising in each stage is very easy. The difference system in the parabolic case will be shown to be stable; consequently, under sufficient hypotheses on the initial and boundary conditions, the solution of the difference equation converges in the mean to that of the heat flow equation. For the elliptic problems, convergence of the iteration will be demonstrated for several types of parameter sequences, and efficient choices of the sequence will be discussed.

Other implicit difference systems have been discussed previously $[2 ; 7]$, along with their relation to explicit equations. The alternating-direction implicit method described in $[2 ; 7]$ is perhaps the most similar to the present scheme. In the two space variable case, the alternating-direction method is probably slightly superior to the procedure to be treated; however, it is not known whether it may be extended to more than two dimensions.

\section{PART I. UnSTEADY-STATE PROBLEMS}

2. Differential and difference systems. We shall treat the first boundary value problem for the heat flow equation in a finite cylinder: $(x, y) \in D$, $0 \leqq t \leqq T$, in the two space variable case; $(x, y, z) \in D, 0 \leqq t \leqq T$, in the threedimensional case. The set $D$ will be assumed to be closed and connected, to have a nonvoid interior, and to have a sufficiently regular boundary in a sense defined below. The following typical notation will be convenient:

Presented to the Society, December 27, 1955; received by the editors December 21, 1955. 


$$
\begin{gathered}
f(i \Delta x, j \Delta y, k \Delta z, n \Delta t)=f_{i j k n}, \\
\Delta_{x}^{2} f_{i j k n}=\left(f_{i+1, j, k, n}-2 f_{i j k n}+f_{i-1, j, k, n}\right) /(\Delta x)^{2} .
\end{gathered}
$$

The subscript $k$ will be suppressed if $z$ is not considered.

It will be convenient to discuss the two-dimensional case first. The boundary value problem then is:

$$
\begin{aligned}
\text { (a) } \frac{\partial^{2} u}{\partial x^{2}}+\frac{\partial^{2} u}{\partial y^{2}}=\frac{\partial u}{\partial t}, \quad(x, y) \in R, 0 \leqq t \leqq T, \\
\text { (b) } u(x, y, 0)=f(x, y), \\
\text { (c) } u(x, y, t)=g(x, y, t),(x, y) \in S, 0 \leqq t \leqq T,
\end{aligned}
$$

where $R$ is nonvoid, open, and connected, $S$ is its boundary, and $D=R \cup S$. Let $D^{*}=D \times[0, T]$; i.e., the set of all $(x, y, t)$ such that $(x, y) \in D$ and $0 \leqq t \leqq T$. We shall consider the following finite difference analogue of (2.2):

$$
\begin{aligned}
& \text { (a) } \Delta_{x}^{2} w_{i, j, n+1}^{*}+\Delta_{y}^{2} w_{i, j, n}=\frac{w_{i, j, n+1}^{*}-w_{i, j, n}}{\Delta t}, \\
& \left((i \Delta x, j \Delta y) \in R^{1}, 0 \leqq n \Delta t \leqq T\right) \\
& \text { (b) } \Delta_{y}^{2} w_{i, i, n+1}=\Delta_{y}^{2} w_{i, j, n}+\frac{w_{i, j, n+1}-w_{i, j, n+1}^{*}}{\Delta t}, \\
& \text { (c) } \quad\left((i \Delta x, j \Delta y) \in R^{1}\right), \\
& \text { (d) } w_{i, j, 0}^{*}=u_{i, j, 0} \quad w_{i, j, n+1}=w_{i, j, n+1}=u_{i, j, n+1}^{1}\left((i \Delta x, j \Delta y) \in S^{1}, 0 \leqq n \Delta t \leqq T\right) .
\end{aligned}
$$

$R^{1}$ is the set of lattice points $(i \Delta x, j \Delta y)$ in $R$ and $S^{1}$ is the set of $(i \Delta x, j \Delta y)$ not in $R$ such that at least one of the points $((i \pm 1) \Delta x, j \Delta y),(i \Delta x,(j \pm 1) \Delta y)$, or $((i \pm 1) \Delta x,(j \pm 1) \Delta y)$ is in $R$. The value $u_{i, j, n+1}^{1}$ is to be taken as the value of $g(x, y, n \Delta t)$ at the nearest point on $S$ to the point of $S^{1}$. It might seem plausible to use the intermediate solution $w_{i, j, n+1}^{*}$ for the solution at $(n+1) \Delta t$; however, this is easily seen to be unstable [2]. Consequently, some "correcting" process must be attached, and we shall see that $(2.3 \mathrm{~b})$ is adequate. No physical significance should be attached to $w_{i, j, n+1}^{*}$; it should be considered only as a first estimate.

For the purpose of computation, equation (2.3) is well adapted; as the linear equations give rise to a Jacobi matrix, simple direct methods for the solution of linear equations of this type exist. In fact, Gaussian elimination works very well [1]. However, in the theoretical analysis to follow a different form is preferable. Eliminating $w_{i, j, n+1}^{*}$ from (2.3a) by means of $(2.3 \mathrm{~b})$,

$$
\begin{array}{r}
\Delta_{x}^{2} w_{i, j, n+1}+\Delta_{y}^{2} w_{i, j, n+1}=\frac{w_{i, j, n+1}-w_{i, j, n}}{\Delta t}+\Delta t \Delta_{x}^{2} \Delta_{y}^{2}\left(w_{i, j, n+1}-w_{i j n}\right) \\
\left((i \Delta x, j \Delta y) \in R^{1}, 0 \leqq n \Delta t \leqq T\right) .
\end{array}
$$


Equation (2.4) is quite similar to the backwards difference equation [2] in that only a term of small magnitude is added. It may also be noted from (2.4) that, while (2.3) appears unsymmetric in $x$ and $y$, it actually is symmetric.

The corresponding difference equation for the three-dimensional problem, involving two intermediate solutions, is
(a) $\Delta_{x}^{2} w_{i, j, k, n+1}^{*}+\Delta_{y}^{2} w_{i j k n}+\Delta_{z}^{2} w_{i j k n}=\frac{w_{i, j, k, n+1}^{*}-w_{i j k n}}{\Delta t}$,
(b) $\Delta_{y}^{2} w_{i, j, k, n+1}^{* *}=\Delta_{y}^{2} w_{i j k n}+\frac{w_{i, j, k, n+1}^{* *}-w_{i, j, k, n+1}^{*}}{\Delta t}$

$$
\left((i \Delta x, j \Delta y, k \Delta z) \in R^{1}, 0 \leqq n \Delta t \leqq T\right),
$$
(c) $\Delta_{z}^{2} w_{i, j, k, n+1}=\Delta_{z}^{2} w_{i j k n}+\frac{w_{i, j, k, n+1}-w_{i, j, k, n+1}^{* *}}{\Delta t}$,
(d) $w_{i, j, k, 0}=u_{i, j, k, 0} \quad\left((i \Delta x, j \Delta y, k \Delta z) \in R^{1}\right)$,
(e) $w_{i, j, k, n+1}^{*}=w_{i, j, k, n+1}^{* *}=w_{i, j, k, n+1}=u_{i, j, k, n+1}^{1}$

$\left((i \Delta x, j \Delta y, k \Delta z) \in S^{1}\right)$.

Again eliminating the intermediate values,

$$
\begin{aligned}
& \Delta_{x}^{2} w_{i, j, k, n+1}+\Delta_{y}^{2} w_{i, j, k, n+1}+\Delta_{z}^{2} w_{i, j, k, n+1} \\
& =\frac{w_{i, j, k, n+1}-w_{i, j, k, n}}{\Delta t}+\Delta t\left(\Delta_{x}^{2} \Delta_{y}^{2}+\Delta_{y}^{2} \Delta_{z}^{2}+\Delta_{z}^{2} \Delta_{x}^{2}\right)\left(w_{i, j, k, n+1}-w_{i j k n}\right) \\
& -(\Delta t)^{2} \Delta_{x}^{2} \Delta_{y}^{2} \Delta_{z}^{2}\left(w_{i, j, k, n+1}-w_{i j k n}\right) \\
& \quad\left((i \Delta x, j \Delta y, k \Delta z) \in R^{1}, 0 \leqq n \Delta t \leqq T\right) .
\end{aligned}
$$

We may now turn to the analysis of the truncation error arising from the use of (2.3) or (2.5). First, a discussion of (2.3) will be given for $R$ a rectangle, which will be taken as a square for arithmetical convenience; then $R$ will be allowed to become more general. The three-dimensional case follows; concluding this part will be a treatment of variable time steps for $g=0$ on $S$. The proofs will be carried out in greater detail in the two-dimensional case in order to simplify some of the arithmetical details, even though the threedimensional problem is more interesting.

3. Error analysis for $R$ a square. Let $R$ be the region $0<x<1,0<y<1$, and let

$$
\Delta x=\Delta y=\frac{1}{N}
$$


It is our object to establish the convergence of $w_{i j n}$ to $u(x, y, t)$ by use of the results of [3], which are based on the concept of a stability analysis. Stability analyses were first described in [9], although the problem of convergence was not treated at length. Essentially, the method consists of combining a timewise superposition principle with the separation of variables technique to determine the growth of the elementary truncation errors caused by replacing derivatives by differences introduced at each grid point. As the points of $S^{\prime}$ fall in $S$, the truncation error vanishes on the boundary; consequently, the stability analysis is performed on the system:

$$
\Delta_{x}^{2} v_{i, j, n+1}+\Delta_{y}^{2} v_{i, j, n+1}=\frac{v_{i, j, n+1}-v_{i, j, n}}{\Delta t}+\Delta t \Delta_{x}^{2} \Delta_{y}^{2}\left(v_{i, j, n+1}-v_{i, j, n}\right)
$$

$$
\begin{array}{lr} 
& (i, j=1, \cdots, N-1), \\
v_{i, j, 0} \text { arbitrary } & (i, j=1, \cdots, N-1), \\
v_{i, j, n}=0 & (i, j=0 \text { or } N) .
\end{array}
$$

The eigenfunctions of (3.2) are of the form

$$
v_{i j n}=\rho_{n} \sin \pi p x_{i} \sin \pi q y_{j}, \quad p, q=1, \cdots, N-1,
$$

where $x_{i}=i \Delta x, y_{j}=j \Delta y$. It is readily seen that

$$
\begin{aligned}
& \frac{\rho_{n+1}}{\rho_{n}} \\
& =\frac{1+\lambda^{2} \sin ^{2}(\pi p / 2 N) \sin ^{2}(\pi q / 2 N)}{1+\lambda\left(\sin ^{2}(\pi p / 2 N)+\sin ^{2}(\pi q / 2 N)\right)+\lambda^{2} \sin ^{2}(\pi q / 2 N) \sin ^{2}(\pi q / 2 N)},
\end{aligned}
$$

where

$$
\lambda=\frac{4 \Delta t}{(\Delta x)^{2}}=\frac{4 \Delta t}{(\Delta y)^{2}} .
$$

Therefore, for any $\Delta t>0,0 \leqq \rho_{n+1} / \rho_{n}<1$, and by definition (2.3) is stable.

THEOREM 1. For $R$ a square, the difference equation (2.3) is stable.

To complete the convergence argument for this case, it is only necessary to establish the magnitude of the elementary truncation errors. Assume that $f(x, y)$ and $g(x, y, t)$ are of such a nature that $u_{x x x x}, u_{y y y y}, u_{t t}$, and $u_{x x y y}$ exist and are bounded in $D^{*}$. Then it is well known [2] that

$$
\Delta_{x}^{2} u_{i, j, n+1}+\Delta_{y}^{2} u_{i, j, n+1}-\frac{u_{i, j, n+1}-u_{i j n}}{\Delta t}=O\left((\Delta x)^{2}+\Delta t\right) .
$$

Moreover, 


$$
\Delta t \Delta_{x}^{2} \Delta_{y}^{2}\left(u_{i, j, n+1}-u_{i j n}\right)=O(\Delta t) .
$$

Consequently,

$$
\begin{array}{r}
\Delta_{x}^{2} u_{i, j, n+1}+\Delta_{y}^{2} u_{i, j, n+1}-\frac{u_{i, j, n+1}-u_{i j n}}{\Delta t}-\Delta t \Delta_{x}^{2} \Delta_{y}^{2}\left(u_{i, j, n+1}-u_{i j n}\right) \\
=O\left((\Delta x)^{2}+\Delta t\right) .
\end{array}
$$

Hence, in the notation of [3], the elementary truncation error $h_{i j n}$ is

$$
h_{i j n}=O\left((\Delta x)^{4}+(\Delta x)^{2} \Delta t\right) .
$$

Thus, by Theorem 1 of [3],

$$
\left\|u_{i j n}-w_{i j n}\right\|=O\left(\frac{(\Delta x)^{4}}{\Delta t}+(\Delta x)^{2}\right)
$$

uniformly in $n$, where

$$
\left\|u_{i j n}-w_{i j n}\right\|=\left\{\frac{1}{N-1} \sum_{i, j=1}^{N-1}\left|u_{i j n}-w_{i j n}\right|^{2}\right\}^{1 / 2} .
$$

Moreover, by Theorem 2 of [3], if $w_{i j n}$ is extended by trilinear interpolation to all of $D^{*}$,

$$
\left\{\int_{D^{*}}|u(x, y, t)|-\left.w(x, y, t)\right|^{2} d V\right\}^{1 / 2}=O\left(\frac{(\Delta x)^{4}}{\Delta t}+(\Delta x)^{2}+(\Delta t)^{2}\right) .
$$

THEOREM 2. If, for a square region, the initial and boundary values of (2.2) are such that the solution of (2.2) possesses $u_{x x x x}, u_{x x y y}, u_{y y, y y}$, and $u_{t t}$ as bounded derivatives in $D^{*}$, then the solution of (2.3) converges in the mean to that of (2.2) with errors given by (3.10) or (3.12) as $\Delta x$ and $\Delta t$ decrease to zero with $(\Delta x)^{4}=o(\Delta t)$.

This completes the discussion of the rectangular region.

4. Generalization. We shall extend the above results to nonrectangular regions in two steps. First, we shall consider domains such that for some sequence with $\left\{\Delta x_{a}\right\}$ with $\Delta x_{a}=\Delta y_{a} \rightarrow 0$ as $a \rightarrow \infty$ the corresponding $S_{a}^{\prime}$ is contained in $S$ for each $a$. Then, more complex boundaries will be treated.

In the first case the stability of the following difference system must be determined:

$$
\Delta_{x}^{2} v_{i, j, n+1}+\Delta_{y}^{2} v_{i, j, n+1}=\frac{v_{i, j, n+1}-v_{i j n}}{\Delta t}+\Delta t \Delta_{x}^{2} \Delta_{y}^{2}\left(v_{i, j, n+1}-v_{i j n}\right)
$$

$$
v_{i, j, n+1}=0
$$$$
\left((i \Delta x, j \Delta y) \in R^{\prime}\right),
$$$$
\left(\left(i \Delta x, j \Delta y, \in S^{\prime}\right)\right) \text {. }
$$ 
Corresponding to (3.3), the eigenfunctions may be assumed to be of the form:

$$
v_{i, j, n}=\rho_{n} \phi_{i j}
$$

Then, if

$$
\begin{array}{cc}
\frac{\rho_{n+1}}{\rho_{n}} & =\mu, \\
\Delta_{x}^{2} \phi_{i j}+\Delta_{y}^{2} \phi_{i j}-\frac{\mu-1}{\mu \Delta t}\left(\phi_{i j}+(\Delta t){ }^{2} \Delta_{x}^{2} \Delta_{y}^{2} \phi_{i j}\right)=0 & \left((i \Delta x, j \Delta y) \in R^{\prime}\right), \\
\phi_{i j}=0 & ((i \Delta x, j \Delta y) \in S) .
\end{array}
$$

For stability, it is necessary that $|\mu| \leqq 1$. In particular, if

$$
\nu=\frac{\mu-1}{\mu \Delta t}
$$

it is sufficient to show that $\nu \leqq 0$.

To facilitate the use of a number of theorems in [6], matrix notation will be introduced. Let $A$ be a matrix corresponding to the operator $\Delta_{x}^{2}+\Delta_{y}^{2}$ and $B$ one corresponding to $\Delta_{x}^{2} \Delta_{y}^{2}$. Then,

$$
A \phi-\nu\left(1+(\Delta t)^{2} B\right) \phi=0 .
$$

Consequently,

$$
\nu=\frac{(A \phi, \phi)}{(\phi, \phi)+(\Delta t)^{2}(B \phi, \phi)}
$$

where

$$
(\phi, \psi)=\frac{1}{N} \sum_{(i \Delta x, j \Delta y) \in R^{\prime}} \phi_{i j} \psi_{i j},
$$

$N$ being the number of points in $R^{\prime}$. The demonstration can be completed by showing that $(A \phi, \phi)<0$ and $(B \phi, \phi)>0$ for any vector $\phi \neq 0$.

Lemma 1. The matrices $A$ and $B$ are symmetric.

Proof. The symmetry of $A(=H)$ is demonstrated in [6, p. 204]. That of $B$ follows from the fact that $B=(K-6 H) /(\Delta x)^{2}(\Delta y)^{2}$ in Milne's notation and the symmetry of $K$ and $H$ [6, p. 212].

LEMMA 2. For rectangular regions, $(A \phi, \phi)<0$ and $(B \phi, \phi)>0$ for all $\phi \neq 0$.

Proof. For rectangular regions, the eigenfunctions for $A$ and $B$ can be taken as $\phi_{p q}=\sin \pi p x / a \sin \pi q y / b, p=1, \cdots, N-1(N \Delta x=a), q=1, \cdots$, 
$M-1(M \Delta x=b)$, where $a$ and $b$ are lengths of the sides of the rectangle. The corresponding eigenvalues $\lambda_{1}$ and $\lambda_{2}$ of $A$ and $B\left(A \phi_{p q}+\lambda_{1} \phi_{p q}=0, B \phi_{p q}\right.$ $\left.+\lambda_{2} \phi_{p q}=0\right)$ are

$$
\begin{aligned}
& \lambda_{1}=\frac{4}{(\Delta x)^{2}}\left[\sin ^{2} \frac{\pi p \Delta x}{2 a}+\sin ^{2} \frac{\pi q \Delta x}{2 b}\right], \\
& \lambda_{2}=-\frac{16}{(\Delta x)^{4}} \sin ^{2} \frac{\pi p \Delta x}{2 a} \sin ^{2} \frac{\pi q \Delta x}{2 b} .
\end{aligned}
$$

The lemma follows immediately from the expansion of $\phi$ in terms of $\left\{\phi_{p q}\right\}$.

THEOREM 3. Let $D$ be a domain such that there exists $\Delta x>0$ such that $S^{\prime}$ is contained in $S$. Then, difference equation (2.3) is stable for this choice of $\Delta x$.

Proof. Let $D^{* *}$ be the least rectangular lattice of mesh widths $\Delta x$ containing the points of $R^{\prime}$ and $S^{\prime}$. Then, the least $\lambda$ corresponding to $A$ applied to $D^{* *}$ is positive and the greatest of $B$ on $D^{* *}$ is negative. By [6, Theorem 3 , p. 164], we may remove the points of $D^{* *}$ exterior to $R^{\prime} \cup S^{\prime}$ one by one without altering this property. Thus, $\nu<0$ for any eigenvalue of (4.6).

THEOREM 4. The conclusions of Theorem 2 hold for a region $D$ such that there exists a sequence $\left\{\Delta x_{a}\right\}, \Delta x_{a} \rightarrow 0$, for which $S_{a}^{\prime} \subset S$ and the remaining hypotheses of Theorem 2 hold.

We are now ready to study the case when $S^{\prime}$ does not lie in $S$. A number of assumptions will be required in the argument below. Let the solution $u(x, y, t)$ of $(2.2)$ be continuable as a solution of $u_{x x}+u_{y y}=u_{t}$ into a domain $E \times[0, T]$, where $R \cup S$ is contained in the interior of $E$, with bounded first derivatives in $E \times[0, T]$. Further, let $\left\{\Delta x_{a}\right\}$ be a null sequence and let $S_{a}^{\prime}$ be the lattice boundary corresponding to $\Delta x_{a}$. Let $S_{a}$ be an analytic closed arc passing through the points of $S_{a}^{\prime}$ and remaining within a distance $2 \Delta x_{a}$ of $S$, and denote by $R_{a}$ the open set interior to $S_{a}$. We may assume that $R_{a}$ contains $R$. Let $g_{a}(x, y, t)$ be an analytic function on $S_{a} \times[0, T]$ interpolating the values $u_{i, j, n+1}^{\prime}$ defined in $\$ 1$. Complete the definition of the initial function $f_{a}(x, y)$ in the region between $S$ and $S_{a}$ so that the solution $u^{(a)}(x, y, t)$ of the problem:

$$
\begin{aligned}
\frac{\partial^{2} u^{(a)}}{\partial x^{2}}+\frac{\partial^{2} u^{(a)}}{\partial y^{2}} & =\frac{\partial u^{(a)}}{\partial t}, \quad(x, y) \in R_{a}, 0 \leqq t \leqq T, \\
u^{(a)}(x, y, 0) & =f_{a}(x, y),(x, y) \in R_{a}, \\
u^{(a)}(x, y, t) & =g_{a}(x, y, t),(x, y) \in S_{a}, \quad 0 \leqq t \leqq T,
\end{aligned}
$$

exists. Moreover, let $f_{a}(x, y)$ vary by $O\left(\Delta x_{a}\right)$ at most from $f(\xi, \eta)$, where $(\xi, \eta)$ is the nearest point on $S$ to $(x, y)$, in the band between $S$ and $S_{a}$. Assume that the $S_{a}, f_{a}(x, y)$, and $g_{a}(x, y, t)$ can be and are chosen such that $u^{(a)}$ can be 
continued to $E \times[0, T]$ with uniformly bounded first derivatives.

Lemma 3. For $(x, y, t) \in R \times[0, T], u(x, y, t)-u^{(a)}(x, y, t)=O\left(\Delta x_{a}\right)$.

Proof. From the construction of $f_{a}(x, y)$ and $g_{a}(x, y, t)$ and the continuation assumption on $u(x, y, t)$ and $u^{(a)}(x, y, t)$, it follows that $u(x, y, t)$ $-u^{(a)}(x, y, t)=O\left(\Delta x_{a}\right)$ on the base and boundary of $R_{a} \times[0, T]$. Application of the maximum principle for parabolic equations (i.e., the solution in the interior of the region is not greater than the maximum of the initial and boundary data) completes the proof.

Lemma 4. For each $(p, q, r)$, there exists a constant $M>0$ such that, for a sufficiently large,

$$
\left|\frac{\partial^{p+q+r} u^{(a)}}{\partial x^{p} \partial y^{q} \partial t^{r}}\right|<M, \quad(x, y, t) \in R_{a} \times[0, T] .
$$

Proof. For $a$ sufficiently large, the distance between the boundary of $E$ and $S_{a}$ is greater than some positive constant. The lemma then follows easily from the Green's function quadrature representation of $u^{(a)}$.

Lemma 5. $\left\|u^{(a)}(x, y, t)-w^{(a)}(x, y, t)\right\|_{L_{\mathbf{2}\left(R_{a}\right)}}=O\left(\left(\Delta x_{a}\right)^{4} / \Delta t_{a}+\left(\Delta x_{a}\right)^{2}+\left(\Delta x_{a}\right)^{2}\right)$.

Proof. This follows from Lemma 4 and Theorem 4 .

Theorem 5. $\left\|u(x, y, t)-w^{(a)}(x, y, t)\right\|_{L_{2}(R)}=O\left(\left(\Delta x_{a}\right)^{4} / \Delta t_{a}+\Delta x_{a}+\left(\Delta t_{a}\right)^{2}\right)$.

Proof. $\left\|u(x, y, t)-w^{(a)}(x, y, t)\right\|_{L_{2}(R)} \leqq\left\|u-u^{(a)}\right\|_{L_{2}(R)}+\left\|u^{(a)}-w^{(a)}\right\|_{L_{2}(R)}$ $\leqq\left\|u-u^{(a)}\right\|_{L_{2}(R)}+\left\|u^{(a)}-w^{(a)}\right\|_{L_{\mathbf{g}}\left(R_{a}\right)}$.

5. Three-dimensional regions. Consider first the unit cube $0<x<1$, $0<y<1,0<z<1$, and let $\Delta x=\Delta y=\Delta z=1 / N$. Then, the error equation becomes:

$$
\begin{aligned}
\left(\Delta_{x}^{2}+\Delta_{y}^{2}+\Delta_{z}^{2}\right) v_{i, j, k, n+1}= & \frac{v_{i, j, k, n+1}-v_{i j k n}}{\Delta t} \\
& +\Delta t\left(\Delta_{x}^{2} \Delta_{y}^{2}+\Delta_{y}^{2} \Delta_{z}^{2}+\Delta_{z}^{2} \Delta_{x}^{2}\right)\left(v_{i, j, k, n+1}-v_{i j k n}\right) \\
& -(\Delta t) \Delta_{x}^{2} \Delta_{y}^{2} \Delta_{z}^{2}\left(v_{i, j, k, n+1}-v_{i j k n}\right) \\
v_{i j k 0} \text { arbitrary } & (i, j, k=1, \cdots, N-1), \\
v_{i, j, k, n+1}=0 & (i, j, k=1, \cdots, N-1), \\
& (i, j, k=0 \text { or } N) .
\end{aligned}
$$

Assuming

$$
v_{i j k n}=\rho_{n} \sin \pi p x \sin \pi q y \sin \pi r z \quad(p, q, r=1, \cdots, N-1)
$$

then 


$$
\begin{aligned}
\frac{\rho_{n+1}}{\rho_{n}}=[1 & +\lambda^{2}\left(\sin ^{2}(\pi p / 2 N) \sin ^{2}(\pi q / 2 N)+\sin ^{2}(\pi q / 2 N) \sin ^{2}(\pi r / 2 N)+\sin ^{2}(\pi r / 2 N) \sin ^{2}(\pi p / 2 N)\right. \\
& \left.+\lambda^{3} \sin ^{2}(\pi p / 2 N) \sin ^{2}(\pi q / 2 N) \sin ^{2}(\pi r / 2 N)\right]\left[1+\lambda\left(\sin ^{2}(\pi q / 2 N)+\sin ^{2}(\pi p / 2 N)\right.\right. \\
& \left.+\sin ^{2}(\pi r / 2 N)\right)+\lambda^{2}\left(\sin ^{2}(\pi p / 2 N) \sin ^{2}(\pi q / 2 N)+\sin ^{2}(\pi q / 2 N) \sin ^{2}(\pi r / 2 N)\right. \\
& \left.+\sin ^{2}(\pi r / 2 N) \sin ^{2}(\pi p / 2 N)+\lambda^{3} \sin ^{2}(\pi p / 2 N) \sin ^{2}(\pi q / 2 N) \sin ^{2}(\pi r / 2 N)\right]^{-1}
\end{aligned}
$$

Thus, (2.5) is stable for any positive time step for a cubic region. It is easy to show that the operators $\Delta_{x}^{2} \Delta_{y}^{2}+\Delta_{y}^{2} \Delta_{z}^{2}+\Delta_{z}^{2} \Delta_{x}^{2}$ and $\Delta_{x}^{2} \Delta_{y}^{2} \Delta_{z}^{2}$ are symmetric; consequently, stability holds in general.

Theorem 6. Difference equation (2.5) is stable.

The obvious analogues of Theorems 2, 4, and 5 can be demonstrated exactly as before and will be omitted.

6. Variable time steps. The boundary conditions for many practical heat flow problems lead to solutions which approach a steady-state condition at late time. As the derivatives of the solution usually decay exponentially with time, it seems reasonable that the time step can be increased. Let $f_{i, j, n}$ denote $f\left(i \Delta x, j \Delta y, t_{n}\right)$, where $t_{0}=0, t_{n}=\sum_{k=0}^{n-1} \Delta t_{k}$. The truncation error equation for the two-dimensional case can be written as follows:

$$
\begin{aligned}
\left(\Delta_{x}^{2}+\Delta_{y}^{2}\right) e_{i, j, n+1}-\frac{e_{i, j, n+1}-e_{i j n}}{\Delta t_{n}} & -\Delta t_{n} \Delta_{x}^{2} \Delta_{y}^{2}\left(e_{i, j, n+1}-e_{i j n}\right) \\
= & \frac{1}{12}\left(\frac{\partial^{4} u}{\partial x^{4}}+\frac{\partial^{4} u}{\partial y^{4}}\right)\left(\Delta x^{2}\right)+\frac{1}{2} \frac{\partial^{2} u}{\partial t^{2}} \Delta t_{n} \\
& -\Delta_{x}^{2} \Delta_{y}^{2}\left(u_{i, j, n+1}-u_{i j n}\right) \Delta t_{n} .
\end{aligned}
$$

Assume that, for some $\delta>0$,

$$
\frac{\partial^{4} u}{\partial x^{4}}, \quad \frac{\partial^{4} u}{\partial y^{4}}, \quad \frac{\partial^{2} u}{\partial t^{2}}, \quad \Delta_{x}^{2} \Delta_{y}^{2}\left(u_{i, j, n+1}-u_{i j n}\right)=O\left(e^{-\delta t_{n}} .\right)
$$

Then, in the terminology of [3],

$$
\left\|h_{i j n}\right\|=O\left(\left((\Delta x)^{4}+(\Delta x)^{2} \Delta t_{n}\right) e^{-\delta t_{n}}\right) .
$$

Moreover, it is readily seen by the methods of [4] that

$$
\left\|e_{i j n}\right\|=O\left(\sum_{k=0}^{n-1}\left\|h_{i j k}\right\|\right) \text {. }
$$

Let the time step grow linearly with the time [4]:

$$
\frac{\Delta t_{n}}{(\Delta x)^{2}}=a+\beta t_{n}
$$


It is easy to see that

$$
t_{n}=\frac{a}{\beta}\left[\left(1+\beta(\Delta x)^{2}\right)^{n}-1\right]
$$

and

$$
\Delta t_{n}=a(\Delta x)^{2}\left(1+\beta(\Delta x)^{2}\right)^{n} .
$$

From [4] and by a similar computation,

$$
\begin{aligned}
\sum_{n=0}^{\infty} \Delta t_{n} e^{-\delta t_{n}} & =O(1), \\
\sum_{n=0}^{\infty} e^{-\delta t_{n}} & =O\left((\Delta \cdot x)^{-2}\right) .
\end{aligned}
$$

Consequently,

$$
\left\|e_{i j n}\right\|=O\left((\Delta x)^{2}\right) .
$$

THEOREM 7. If (6.2) is valid, then the solution of (2.3) with time steps given by (5.5) converges in the mean to that of (2.2) with the error being $O\left((\Delta x)^{2}\right)$.

Obviously, the analogous theorem in the three-dimensional case is valid. This completes the discussion of the unsteady-state problems.

\section{Part II. Steady-state PRoblems}

7. Iteration method. The Dirichlet problem for Poisson's equation

$$
\begin{aligned}
\frac{\partial^{2} u}{\partial x^{2}}+\frac{\partial^{2} u}{\partial y^{2}} & =f(x, y), & & (x, y) \in R, \\
u(x, y) & =g(x, y), & & (x, y) \in S,
\end{aligned}
$$

or

$$
\begin{array}{rlrl}
\frac{\partial^{2} u}{\partial x^{2}}+\frac{\partial^{2} u}{\partial y^{2}}+\frac{\partial^{2} u}{\partial z^{2}} & =f(x, y, z), & & (x, y, z) \in R, \\
u(x, y, z) & =g(x, y, z), & (x, y, z) \in S,
\end{array}
$$

will be studied. As for the heat flow equation, the differential equation will be replaced by a finite difference equation. However, there will be a fundamental difference in outlook between the two types of problems. In the unsteadystate case, the principal question was that of convergence of the solution of a proposed difference equation to that of the differential equation. For the steady-state problem with boundary data liable to arise from physical problems, well known difference formulae exist for its numerical solution giving 
any desired accuracy; however, the difference equation to be treated leads to a large system of linear equations to be solved. Thus, the major problem in this case is the actual evaluation of the numerical solution, having chosen the net size in advance.

Let us discuss first the two-dimensional case. The following difference analogue of (7.1) will be studied:

$$
\begin{aligned}
\Delta_{x}^{2} w_{i j}+\Delta_{y}^{2} w_{i j} & =f_{i j}, & & (i \Delta x, j \Delta y) \in R^{\prime}, \\
w_{i j} & =u_{i j}, & & (i \Delta x, j \Delta y) \in S^{\prime} .
\end{aligned}
$$

Numerous proofs (a bibliography is given in [6]) have been given showing that $u_{i j}$ converges to the solution $u(x, y)$ under quite general boundary conditions. It is assumed that here $f(x, y)$ and $g(x, y)$ are sufficiently well behaved and $\Delta x$ is fixed small enough so that $w_{i j}$ approximates $u(x, y)$ to the required accuracy.

The solution of the linear equations (7.3) may be accomplished by either some direct method or an iterative procedure. Experience has shown that direct methods such as elimination are quite lengthy, whereas several iterative methods have proved capable of reducing the total calculation by large factor. Many of these procedures are easily seen to be closely related to methods for solving the unsteady-state problem [5]. It is the object of this chapter to adapt difference equation (2.3) to the iterative solution of (7.3), and similarly for (2.5) for the three-dimensional problem.

Let $w_{i j}^{(0)}$ be an arbitrary mesh function agreeing with $u(x, y)$ on the boundary of the region. Then, let, for $n \geqq 0$,

$$
\begin{aligned}
\Delta_{x}^{2} w_{i j}^{(n+1 / 2)}+\Delta_{y}^{2} w_{i j}^{(n)} & =\frac{w_{i j}^{(n+1 / 2)}-w_{i j}^{(n)}}{a_{n}}+f_{i j}, & & (i \Delta x, j \Delta y) \in R^{\prime}, \\
\Delta_{y}^{2} w_{i j}^{(n+1)} & =\Delta_{y}^{2} w_{i j}^{(n)}+\frac{w_{i j}^{(n+1)}-w_{i j}^{(n+1 / 2)}}{a_{n}}, & & (i \Delta x, j \Delta y) \in R^{\prime}, \\
w_{i j}^{(n)} & =u_{i j}, & & (i \Delta x, j \Delta y) \in S^{\prime},
\end{aligned}
$$

where the parameter sequence $\left\{a_{n}\right\}$ will be chosen later.

The equations in this form are, as in the parabolic case, suitable for the numerical calculation but not amenable to the mathematical analysis. Obviously, the intermediate values $w_{i j}^{(n+1 / 2)}$ can be eliminated with the resulting equation being

$$
\begin{aligned}
& \Delta_{x}^{2} w_{i j}^{(n+1)}+\Delta_{y}^{2} w_{i j}^{(n+1)}=\frac{w_{i j}^{(n+1)}-w_{i j}^{(n)}}{a_{n}}+a_{n} \Delta_{x}^{2} \Delta_{y}^{2}\left(w_{i j}^{(n+1)}-w_{i j}^{(n)}\right)+f_{i j}, \\
& w_{i j}=u_{i j},(i \Delta x, j \Delta y) \in R^{\prime}, \\
&(i \Delta x, j \Delta y) \in S^{\prime} .
\end{aligned}
$$


It will again be convenient to treat the case of a rectangular domain first and then generalize to more complex regions. The three-dimensional problem follows.

8. Convergence of the iteration for rectangular domain. Let

$$
v_{i j}^{(n)}=w_{i j}-w_{i j}^{(n)} .
$$

Then, the residual error $v_{i j}^{(n)}$ after $n$ iterations satisfies the difference equation

$$
\Delta_{x}^{2} v_{i j}^{(n+1)}+\Delta_{y}^{2} v_{i j}^{(n+1)}=\frac{v_{i j}^{(n+1)}-v_{i j}^{(n)}}{a_{n}}+a_{n} \Delta_{x}^{2} \Delta_{y}^{2}\left(v_{i j}^{(n+1)}-v_{i j}^{(n)}\right),
$$

$$
\begin{array}{ll}
v_{i j}^{(n)}=0, & (i \Delta x, j \Delta y) \in R^{\prime}, \\
& (i \Delta x, j \Delta y) \in S^{\prime} .
\end{array}
$$

Let

$$
\lambda_{n}=\frac{4 a_{n}}{(\Delta x)^{2}}
$$

and

$$
\rho_{p q}\left(\lambda_{n}\right)=\frac{1+\lambda_{n}^{2} \sin ^{2} \pi p / 2 N \sin ^{2} \pi q / 2 N}{1+\lambda_{n}\left(\sin ^{2} \pi p / 2 N+\sin ^{2} \pi q / 2 N\right)+\lambda_{n}^{2} \sin ^{2} \pi p / 2 N \sin ^{2} \pi q / 2 N} .
$$

As $v_{i j}^{(n)}$ can be expanded in a double Fourier series,

$$
v_{i j}^{(n)}=\sum_{p, q=1}^{N-1} c_{p q}^{(n)} \sin \pi p x \sin \pi q y .
$$

From (3.4)

$$
v_{i j}^{(n+1)}=\sum_{p, q=1}^{N-1} c_{p q}^{(n)} \rho_{p q}\left(\lambda_{n}\right) \sin \pi p x \sin \pi q y
$$

Hence,

$$
v_{i i}^{(n)}=\sum_{p, q=1}^{N-1} c_{p q}^{(0)}\left(\prod_{k=0}^{n-1} \rho_{p q}\left(\lambda_{k}\right)\right) \sin \pi p x \sin \pi q y .
$$

Restrict the sequence $\left\{a_{n}\right\}$ to be made up of numbers which are positive or purely imaginary and which, moreover, satisfy

$$
0<M_{1}<\left|a_{n}\right|<M_{2}<\infty, \quad n \geqq 0,
$$

for some arbitrary constants $M_{1}$ and $M_{2}$. It is apparent from (8.4) that there exists $\epsilon>0$ such that

$$
\left|\rho_{p q}\left(\lambda_{n}\right)\right|<1-\epsilon, \quad 1 \leqq p, q \leqq N-1, n \geqq 0 .
$$


Therefore,

$$
\left\|v_{i j}^{(n)}\right\|=\left\{\sum_{p, q=1}^{N-1}\left|c_{p q}^{(0)}\right|^{2} \prod_{k=0}^{n-1}\left|\rho_{p q}\left(\lambda_{k}\right)\right|^{2}\right\}^{1 / 2}<(1-\epsilon)^{n}\left\|v_{i j}^{(0)}\right\| .
$$

The following theorem has been proved:

THEOREM 8. If $\left\{a_{n}\right\}$ is any sequence consisting only of positive and pure imaginary numbers which are bounded in modulus away from zero and infinity, then $w_{i j}^{(n)}$ as defined by (7.4) converges to the solution $w_{i j}$ of (7.3).

Convergence has been proved for a rather wide class of sequences $\left\{a_{n}\right\}$. However, the rate of convergence is not independent of the choice of these iteration constants; the next three sections of this chapter will be devoted to determining efficient choices for planar domains of this sequence to minimize the total calculation effort to reduce the residual to a preassigned level of smallness.

9. Real sequences, square domain. The simplest approach is to set up a finite sequence of constants each reducing one particular term in the expansion (8.5) of the residual as much as possible, and then repeat this sequence until the error is damped out. Consider the function

$$
\rho=\rho(\lambda)=\frac{1+\lambda^{2} \xi \eta}{1+\lambda(\xi+\eta)+\lambda^{2} \xi \eta},
$$

where $\xi=\sin ^{2} \pi p / 2 N$ and $\eta=\sin ^{2} \pi q / 2 N$ are held fixed. Then,

$$
\frac{d \rho}{d \lambda}=\frac{\xi+\eta}{\left[1+\lambda(\xi+\eta)+\lambda^{2} \xi \eta\right]^{2}}\left(\lambda^{2} \xi \eta-1\right)
$$

Hence, the minimum value of $\rho$ occurs when

$$
\lambda=\frac{1}{(\xi \eta)^{1 / 2}}
$$

and this value is

$$
\rho_{\min }=\frac{1}{1+(\xi+\eta) / 2(\xi \eta)^{1 / 2}} .
$$

Moreover, it is easy to see that

$$
\max _{\xi, \eta} \rho_{\min }=\frac{1}{2}
$$

and that whenever $\xi=\eta$ the value one-half is assumed by $\rho_{\text {min }}$.

Thus, if 
(9.6) $a_{p q}=\frac{\lambda_{p q}(\Delta x)^{2}}{4}=\frac{1}{4 N^{2} \sin \pi p / 2 N \sin \pi q / 2 N}, p, q=1, \cdots, N-1, p \leqq q$, and the iteration proceeds through these $N(N-1) / 2$ steps with constants $\left\{a_{p q}\right\}$, then the modulus of each coefficient in the error is reduced to a value not larger than one half of its initial value. The one half is too conservative, as this takes into account the effect of only one iteration on each term. Consider an iteration using an iteration parameter corresponding to a principal diagonal term as it affects an off-diagonal term having one index equal to that of the diagonal index. Let

$$
\lambda_{p p}=\frac{1}{\sin ^{2}(\pi p / 2 N)}
$$

Then,

$$
\rho_{p q}\left(\lambda_{p p}\right)=1 / 2 .
$$

Hence, if only the $N-1$ iterations corresponding to $\lambda_{11}, \lambda_{22}, \cdots, \lambda_{N-1, N-1}$ are taken, the magnitude of each error component is at least halved. Further grouping of terms can also lead to a reduction in work. The following lemma is helpful.

Lemma 6. If $\lambda$ is a positive number,

$$
\rho_{p q}(\lambda)^{2} \leqq \rho_{p p}(\lambda) \rho_{q q}(\lambda) .
$$

Proof. Let

$$
\xi_{p}=\sin ^{2} \frac{\pi p}{2 N}
$$

Then,

$$
\begin{aligned}
\rho_{p q}^{2} & =\frac{1+2 \lambda^{2} \xi_{p} \xi_{q}+\lambda^{4} \xi_{p}^{2} \xi_{q}^{2}}{\left(1+\lambda \xi_{p}\right)^{2}\left(1+\lambda \xi_{q}\right)^{2}} \\
& \leqq \frac{1+\lambda^{2}\left(\xi_{p}^{2}+\xi_{q}^{2}\right)+\lambda^{4} \xi_{p}^{2} \xi_{q}^{2}}{\left(1+\lambda \xi_{p}\right)^{2}\left(1+\lambda \xi_{q}\right)^{2}} \text { as } 2 \xi_{p} \xi_{q} \leqq \xi_{p}^{2}+\xi_{q}^{2} \\
& =\rho_{p p \rho} \rho_{q q} .
\end{aligned}
$$

An immediate corollary of this lemma is the following: if there exists a sequence $\left\{\lambda_{k}\right\}_{1}^{M}$ of real numbers such that, for each $p$, there corresponds at least one $k$ such that $\rho_{p p}\left(\lambda_{k}\right) \leqq 1 / 2+\epsilon$, then after the $M$ iterations corresponding to $\left\{\lambda_{k}\right\}$ each error component will have been reduced by a factor of $1 / 2+\epsilon$ at least, as 


$$
\prod_{k=1}^{M} \rho_{p q}\left(\lambda_{k}\right)^{2} \leqq \prod_{k=1}^{M} \rho_{p p}\left(\lambda_{k}\right) \prod_{k=1}^{M} \rho_{q q}\left(\lambda_{k}\right) \leqq(1 / 2+\epsilon)^{2} .
$$

As a consequence of this result, the study will be limited to obtaining sequences such as these. Let

$$
\lambda_{k}=1 / \eta_{k}
$$$$
k=1,2, \cdots, M,
$$

and

$$
z=\xi_{p} / \eta_{k}
$$

Then,

$$
\rho_{p p}\left(\lambda_{k}\right)=\frac{1+z^{2}}{(1+z)^{2}} .
$$

If $\rho_{p p}\left(\lambda_{k}\right) \leqq 1 / 2+\epsilon$, then it is easy to see that

$$
\frac{1+2 \epsilon-(8 \epsilon)^{1 / 2}}{1-2 \epsilon} \leqq z \leqq \frac{1+2 \epsilon+(8 \epsilon)^{1 / 2}}{1-2 \epsilon} .
$$

If the $\eta_{k}$ are chosen so that

$$
\frac{1+2 \epsilon+(8 \epsilon)^{1 / 2}}{1-2 \epsilon} \eta_{k}=\frac{1+2 \epsilon-(8 \epsilon)^{1 / 2}}{1-2 \epsilon} \eta_{k+1},
$$

and

$$
\begin{aligned}
\eta_{1} & =\xi_{1}, \\
\eta_{M} & \geqq 1, \quad \eta_{M-1}<1,
\end{aligned}
$$

then for each $p$ there is either one or two values of $k, 1 \leqq k \leqq M$, such that $\rho_{p p}\left(\lambda_{k}\right) \leqq 1 / 2+\epsilon$. Solving (9.17) and (9.18) for $\eta_{k}$,

$$
\eta_{k}=\left(\frac{1+2 \epsilon+(8 \epsilon)^{1 / 2}}{1+2 \epsilon-(8 \epsilon)^{1 / 2}}\right)^{k-1} \xi_{1}, \quad k=1, \cdots, M .
$$

The number $M$ may be found as below:

$$
\begin{aligned}
(M-2) \log \frac{1+2 \epsilon-(8 \epsilon)^{1 / 2}}{1+2 \epsilon+(8 \epsilon)^{1 / 2}} & <-\log \sin ^{2} \frac{\pi}{2 N} \\
& \leqq(M-1) \log \frac{1+2 \epsilon+(8 \epsilon)^{1 / 2}}{1+2 \epsilon-(8 \epsilon)^{1 / 2}} .
\end{aligned}
$$

Thus, for large $N$,

$$
M \sim \frac{2 \log (2 N / \pi)}{\log \left(\left(1+2 \epsilon+(8 \epsilon)^{1 / 2}\right) /\left(1+2 \epsilon-(8 \epsilon)^{1 / 2}\right)\right)} .
$$


It will be informative to compute the total amount of computation necessary to complete the solution to a desired degree of error reduction and then to determine the optimum choice of $\epsilon$.

Using the Gaussian elimination method to solve the equations of (7.4), it takes $20(N-1)^{2}$ calculations to complete one iteration. Thus, to reduce the error components to $1 / 2+\epsilon$ of their original magnitudes,

$$
40(N-1)^{2} \log \frac{2 N}{\pi} / \log \frac{1+2 \epsilon+(8 \epsilon)^{1 / 2}}{1+2 \epsilon-(8 \epsilon)^{1 / 2}}
$$

calculations are required. If it is desired that the reduction be $\exp (-Q)$, then the total work will be

$$
W=-40 Q(N-1)^{2} \log \frac{2 N}{\pi} / \log (1 / 2+\epsilon) \log \frac{1+2 \epsilon+(8 \epsilon)^{1 / 2}}{1+2 \epsilon-(8 \epsilon)^{1 / 2}}
$$

$W$ is minimized as a function of $\epsilon$ for $\epsilon$ about 0.18 , and this minimum work is about

$$
W_{\min }=37 Q(N-1)^{2} \log \frac{2 N}{\pi} .
$$

This is superior to the "modified Liebmann" method (over-relaxation) $[5 ; 8]$, which is $O\left(N^{3}\right)$, but slightly inferior to the alternating-direction implicit method [7], which is of the same order as this procedure with a smaller constant than 37 .

10. Imaginary sequences, square domain. A sequence of purely imaginary numbers can be determined such that an exact solution of (7.3) is obtained after a finite number of iterations. Let

$$
\lambda_{p q}=\frac{i}{\sin (\pi p / 2 N) \sin (\pi q / 2 N)}, \quad p, q=1, \cdots, N-1 .
$$

Then,

$$
\rho_{p q}\left(\lambda_{p q}\right)=\rho_{q p}\left(\lambda_{p q}\right)=0,
$$

and, consequently, after iterating with the $(N-1) N / 2$ different values of $\lambda_{p q}$ all error components will have been eliminated. Note, however, that this requires $O\left(N^{4}\right)$ calculations, which is considerably more than that necessary to obtain a good approximation to the solution by iteration using a real sequence or, as to be shown below, an imaginary sequence.

Lemma 6 fails to hold for other than real $\lambda$; the following lemma will replace it and can be demonstrated simply.

Lemma 7. If $(1+\epsilon)^{-1} \leqq \sin ^{2}(\pi p / 2 N) \sin ^{2}(\pi q / 2 N) / \sin ^{4}(\pi k / 2 N) \leqq(1-\epsilon)^{-1}$, then $\left|\rho_{p q}\left(\lambda_{k k}\right)\right| \leqq \epsilon$. 
This lemma again allows us to choose a sequence of iteration parameters corresponding to diagonal elements. Again the number of iterations in one cycle is $O(\log N)$. The minimum work can be computed to be about

$$
W_{\min }=102 Q(N-1)^{2} \log \frac{2 N}{\pi} .
$$

As this is inferior to the result with real sequences, imaginary sequences provide only another essentially impractical direct method of solving exactly the Laplace difference equation.

11. General region for two-dimensional problem. As we are treating only the solution of the linear equations, the assignment of the boundary values for the difference equation plays no role in our discussion. Also, only real parameter sequences will be considered.

Let the region be contained in a rectangle of side lengths $N_{1} \Delta x$ and $N_{2} \Delta x$, $N_{1} \geqq N_{2}$. Then, one admissible choice for the parameter sequence is that sequence developed for iterating on the square of side length $N_{1} \Delta x$. By the inclusion theorem [6, Theorem 3, p. 164] on eigenvalues used in the proof of Theorem 3, it is obvious that the coefficient of each eigenvector in the general case is reduced as before. Consequently,

$$
W_{\min } \leqq 37 Q\left(N_{1}-1\right)\left(N_{2}-1\right) \log \frac{2 N}{\pi} .
$$

In fact, if $s$ is the number of interior points in the lattice,

$$
W_{\min } \leqq 37 Q s \log \frac{2 N}{\pi} .
$$

While undoubtedly the parameter sequence chosen above is not the most efficient, it may not be worth the effort in a practical problem to obtain a better one.

12. Three-dimensional problem. The iterative analogue of $(2.5)$ is the following:

$$
\begin{aligned}
& \Delta_{x}^{2} w_{i, j, k}^{(n+1 / 3)}+\Delta_{y}^{2} w_{i, j, k}^{(n)}+\Delta_{z}^{2} w_{i, j, k}^{(n)}=\frac{w_{i, j, k}^{(n+1 / 3)}-w_{i, j, k}^{(n)}}{a_{n}}+f_{i j k}, \\
& \Delta_{y}^{2} w_{i, j, k}^{(n+2 / 3)}=\Delta_{y}^{2} w_{i, j, k}^{(n)}+\frac{w_{i, j, k}^{(n+2 / 3)}-w_{i, j, k}^{(n+1 / 3)}}{a_{n}} \quad(i \Delta x, j \Delta x, k \Delta x) \in R^{\prime}, \\
& \Delta_{z}^{2} w_{i, j, k}=\Delta_{z}^{2} w_{i, j, k}^{(n)}+\frac{w_{i, j, k}^{(n+1)}-w_{i, j, k}^{(n+2 / 3)}}{a_{n}}, \\
& w_{i, j, k}^{(n+1 / 3)}=w_{i, j, k}^{(n+2 / 3)}=w_{i, j, k}^{(n+1)}=u_{i, j, k} \quad(i \Delta x, j \Delta x, k \Delta x) \in S^{\prime} .
\end{aligned}
$$


As in the two-dimensional problem, it is sufficient to discuss the case of a unit cubic region only. Let

$$
\rho_{p q r}(\lambda)=1-\frac{\lambda\left(\xi_{p}+\xi_{q}+\xi_{r}\right)}{\left(1+\lambda \xi_{p}\right)\left(1+\lambda \xi_{q}\right)\left(1+\lambda \xi_{r}\right)} .
$$

It is easy to see that for $p=q=r$

$$
\min _{\lambda>0} \rho_{p p p}(\lambda)=\rho\left(1 / 2 \xi_{p}\right)=5 / 9 .
$$

It is again possible to group the eigenfunctions so as to reduce the number of iterations necessary. Consider $\lambda_{k}=1 / 2 \xi_{k}^{*}$ and let $a=\xi_{p} / \xi_{k}{ }^{*}, b=\xi_{q} / \xi_{k}{ }^{*}$, and $c=\xi_{r} / \xi_{k}^{*}$. Then $\rho_{p q r}(\lambda)$ becomes

$$
f(a, b, c)=1-\frac{1}{2} \frac{a+b+c}{(1+a / 2)(1+b / 2)(1+c / 2)} .
$$

Let $(a, b, c)$ satisfy any of the following relations:
(a) $\alpha \leqq a \leqq \beta$,
$0 \leqq b \leqq \beta$,
$0 \leqq c \leqq \beta$,
(b) $0 \leqq a \leqq \beta$,
$\alpha \leqq b \leqq \beta$,
$0 \leqq c \leqq \beta$,
(c) $0 \leqq a \leqq \beta$,
$0 \leqq b \leqq \beta$,
$\alpha \leqq c \leqq \beta$,

where $0<\alpha<1, \beta>1$. Then the maximum of $f(a, b, c)$ in this range is either $f(\alpha, 0,0)$ or $f(\beta, \beta, \beta)$. For greatest efficiency these values should be the same; in this case

$$
\begin{aligned}
\alpha & =\frac{24 \beta}{(2+\beta)^{3}-12 \beta}, \\
f(\alpha, 0,0) & =f(\beta, \beta, \beta)=1-12 \beta(2+\beta)^{-3} .
\end{aligned}
$$

If a cycle of iterations is made using the sequence $\left\{\lambda_{k}\right\}$ corresponding to$$
\xi_{k}^{*}=(\alpha / \beta)^{k}=\left[24\left\{(2+\beta)^{3}-12 \beta\right\}^{-1}\right]^{k}, \quad k=0, \cdots, M,
$$

where $\xi_{M-1}^{*}>\sin ^{2} \pi / 2 N, \xi_{M}^{*} \leqq \sin ^{2} \pi / 2 N$, then each $\left(\xi_{p}, \xi_{q}, \xi_{r}\right)$ satisfies (12.5) for at least one iteration in the cycle. Thus, the magnitude of the coefficient of each eigenfunction is reduced by a factor of $f(\beta, \beta, \beta)$ at least.

To determine the work required to reduce the error by $\exp (-Q)$, it is necessary to obtain the number of calculations per iteration, the number of iterations in a cycle, and the number of cycles used. If the method of [1] is used again, $31(\mathrm{~N}-1)^{3}$ operations are required per iteration. The number of iterations per cycle is about

$$
2 \log \frac{2 N}{\pi} / \log \frac{(2+\beta)^{3}-12 \beta}{24},
$$


and the number of cycles is about

$$
Q /-\log \left(1-12 \beta(2+\beta)^{-3}\right) \text {. }
$$

Thus, the total number $W$ of calculations is

(12.8) $W=12 Q(N-1)^{3} \log \frac{2 N}{\pi} /-\log \left(1-12 \beta(2+\beta)^{-3}\right) \log \frac{(2+\beta)^{3}-12 \beta}{24}$.

$W$ is minimized as a function of $\beta$ for $\beta$ around four. For this choice of $\beta$,

$$
W_{\min }=127 Q(N-1)^{3} \log \frac{2 N}{\pi} .
$$

The application of the method to general regions is exactly as for the twodimensional case. Again letting $R$ be contained in a cube of length $N_{1} \Delta x$ on a side and letting $s$ be the number of interior lattice points,

$$
W_{\min }=127 Q s \log \frac{2 N_{1}}{\pi} .
$$

As the best possible order of $W$ is $O\left(N^{3}\right)$, this method is quite satisfactory on this point. The successive over-relaxation method [8] requires $O\left(N^{4}\right)$ calculations and is, consequently, not as efficient for large $N$. Undoubtedly, there exist yet undiscovered procedures that can improve on the coefficient 127.

\section{REFERENCES}

1. G. H. Bruce, D. W. Peaceman, H. H. Rachford, and J. D. Rice, Calculations of unsteadystate gas flow through porous media, Trans. Amer. Inst. Mining Metallurgical Engrs. vol. 198 (1953) pp. 79-91.

2. J. Douglas, On the numerical integration of $u_{x x}+u_{y y}=u_{t}$ by implicit methods, J. Soc. Ind. Appl. Math. vol. 3 (1955) pp. 42-65.

3. - On the relation between stability and convergence in the numerical solution of linear parabolic and hyperbolic differential equations, to appear in J. Soc. Ind. Appl. Math. vol. 4.

4. J. Douglas and T. M. Gallie, Variable time steps in the solution of the heat flow equation by a difference equation, Proc. Amer. Math. Soc. vol. 6 (1955) pp. 787-793.

5. S. P. Frankel, Convergence rates of iterative treatments of partial differential equations, Math. Tables and Other Aids to Computation vol. 4 (1950) pp. 65-75.

6. W. E. Milne. Numerical solution of differential equations, New York, 1953.

7. D. W. Peaceman and H. H. Rachford, The numerical solution of parabolic and elliptic differential equations, J. Soc. Ind. Appl. Math. vol. 3 (1953) pp. 28-41.

8. D. Young, Iterative methods for solving partial difference equations of elliptic type, Trans. Amer. Math. Soc. vol. 76 (1954) pp. 92-111.

9. G. G. O'Brien, M. A. Hyman and S. Kaplan, A study of the numerical solution of partial differential equations, Journal of Mathematics and Physics vol. 29 (1951) pp. 223-251.

Humble Oil and Refining Co., Houston, TeX. 